\title{
The unique ethical challenges of conducting research in the rehabilitation medicine population Jeff Blackmer*
}

Address: Division of Physical Medicine and Rehabilitation University of Ottawa The Rehabilitation Centre 505 Smyth Road Ottawa, ON K1H 8M2

Email: Jeff Blackmer* - jblackmer@ottawahospital.on.ca

* Corresponding author

Published: 17 June 2003

BMC Medical Ethics 2003, 4:2
Received: 19 February 2003

Accepted: 17 June 2003

This article is available from: http://www.biomedcentral.com/I472-6939/4/2

(C) 2003 Blackmer; licensee BioMed Central Ltd. This is an Open Access article: verbatim copying and redistribution of this article are permitted in all media for any purpose, provided this notice is preserved along with the article's original URL.

\begin{abstract}
Background: The broad topic of research ethics is one which has been relatively well-investigated and discussed. Unique ethical issues have been identified for such populations as pediatrics, where the issues of consent and assent have received much attention, and obstetrics, with concerns such as the potential for research to cause harm to the fetus. However, little has been written about ethical concerns which are relatively unique to the population of patients seen by the practitioner of rehabilitation medicine.

Discussion: This paper reviews unique ethical concerns in conducting research in this population, including decision-making capacity, communication, the potential for subject overuse, the timing of recruitment, hope for a cure and therapeutic misconception and the nature of the health care provider-research subject relationship.

Summary: Researchers in the area of rehabilitation medicine should be aware of some of the unique ethical challenges posed by this patient population and should take steps to address any potential concerns in order to optimize subject safety and ensure that studies meet current ethical guidelines and standards.
\end{abstract}

\section{Background}

In each medical specialty, there are various patient characteristics that have the potential to present unique ethical and practical challenges in conducting research in that specialty. In pediatrics, the issues might include such things as patient age precluding truly informed consent, substitute decision making by the parents, or the issue of patient assent versus consent [1]. In obstetrics research, one must carefully consider potential benefits and harms to the fetus while respecting the rights and wishes of the mother [2-4]. In medical genetics, there are potential implications of research results not only for the participant but also for family members and future progeny [5].

All these issues, and these particular patient populations, have been extensively studied and discussed in the subspecialty and ethics literature. However, the subject of unique issues in research ethics in the rehabilitation patient population has not been investigated to any significant degree. A literature search, conducted through the use of Medline and Bioethicsline (please note that Bioethicsline is now retired and replaced by NLM gateway at 
http:/(gateway.nlm.nih.gov) in this area revealed no articles or publications regarding this topic.

The objective of this paper is to identify and discuss certain issues which might be considered relatively unique to rehabilitation medicine. These include decision-making capacity (which includes both the concepts of capacity and competency), communication and language, the potential for overuse of individual research subjects, problems with the timing of subject recruitment, the hope for a cure in certain patient populations and the nature of the health care provider - research subject relationship in the rehabilitation setting. Each will be discussed separately.

\section{Discussion}

\section{Decision-making capacity}

It is widely recognized that in order to consent to participate in a research protocol, three conditions must be met [6] - patient capacity (the ability of the patient to understand the nature of the research, as well as its risks and benefits, in order to make an informed decision), voluntariness (freedom from undue coercion, be it deliberate or unintended) and disclosure (the provision of all information necessary for the potential subject to assist them in the decision-making process).

There are many different types of patients and patient diagnostic categories treated within the specialty of rehabilitation medicine. Some patients have isolated deficits such as multiple fractures or spinal cord injuries with no involvement of the brain or of cognitive function, and in this case, decision-making capacity is generally not a significant factor which needs to be considered in the process of obtaining informed consent. However, a fairly large proportion of patients seen by rehabilitation medicine practitioners may have cognitive deficits, and these deficits can range in severity from minor and subtle to significant and overt.

The population of patients who have suffered a traumatic brain injury (TBI) is a good example. The severity of TBI can range from mild to severe. Accordingly, persistent cognitive deficits can range from mild and temporary to severe and persistent. In cases of severe TBI, it may be obvious that the patient does not have sufficient decisionmaking capacity to be able to decide whether or not to participate in a research protocol, and alternative arrangements (such as proxy consent from a member of the family) may have to be sought. In the case of more subtle deficits, further capacity assessment may be required to ascertain a particular individual's level of decision-making ability.

Stroke is the most common diagnosis among patients in most rehabilitation medicine programs [7]. Stroke patients subsequently make up a large proportion of potential research candidates, both as inpatients and outpatients. Although a fairly significant number of stroke patients may recover neurologically to the point where they do not have any cognitive deficits which would interfere with the decision-making process, many patients will continue to have damage to the parts of the brain involved in judgment and reasoning, and these patients might not be able to make a truly informed decision regarding participation in a research protocol. Again, if a concern exists, the patient may need to undergo further capacity assessment.

Two other populations frequently seen by rehabilitation medicine practitioners are patients with multiple sclerosis (MS) and those with Parkinson's disease (PD). These patients often have very obvious physical impairments and limitations (such as tremor, weakness and incoordination) that tend to receive the majority of medical and therapeutic attention. However, a very significant proportion of patients in each group will go on to develop cognitive deficits that may affect decision-making capacity but might be quite subtle unless specifically tested for $[8,9]$. Both groups commonly develop what is termed a subcortical-type dementia. Unlike the more common Alzheimer's-type dementia, which manifests itself primarily with deficits in memory and environmental awareness, the deficits in subcortical dementias are less apparent. These deficits often present as difficulties with higher-level cognitive functions such as reasoning, insight, judgment and abstraction. These difficulties, of course, are relevant to capacity and may be present in a degree sufficient to interfere with a patient's ability to decide whether or not to participate in a research protocol, but unless specifically tested for, will not always be apparent to the person obtaining informed consent.

There are many different types of patients seen by rehabilitation practitioners where decision-making capacity may be of concern in the process of attempting to obtain informed consent. Since it may be particularly difficult in some of these cases to know whether a prospective subject has adequately understood the relevant information and appreciated the potential harms and benefits of participating, the researcher must be vigilant and willing to explore any 'red flags' that may indicate lack of sufficient decisionmaking capacity (arranging for a capacity assessment if necessary) in order to ensure the participation of the subject is truly informed and voluntary.

\section{Communication issues}

In order to be able to consent to participate in a research study, the potential subject must be able to understand the nature and specific content of the protocol, as well as the risks and benefits which are involved in participation. 
In order to be a subject, the patient will generally also need to be able to communicate with the researcher. Both these areas can be impaired in patients with language deficits.

The ability to use language to communicate with others, be it in a written or verbal (or other) form, requires the ability to comprehend information and to present information to others in a form that they can understand. Many patients in the rehabilitation population may have varying degrees and types of aphasia.

If the language deficit involves comprehension, then informed consent will obviously be difficult to obtain from that particular subject, and they may have to be excluded from the study, or proxy consent sought. For patients with expressive deficits, they may be able to provide consent through non-verbal communication, but it may be very difficult for them to participate in the research protocol unless specific mechanisms are in place to accommodate their deficits.

For example, some patients might substitute one word for another when they attempt to express themselves. In some cases the new word may be a nonsense word (or neologism) and it will be obvious to the researcher that the answer is not valid. However, some patients will substitute a true word, but one which has a different meaning then they wish to express. Some patients will the use the word "yes" in place of the word "no" because of their aphasia, and this substitution will obviously have significant repercussions on the ability of the researcher to obtain valid and accurate data.

Therefore, in situations where a potential research subject is thought to have aphasia, a thorough assessment should be performed (usually by a Speech and Language Pathologist) to see if the patient is not only able to consent to participation, but whether or not their communication skills are sufficient to permit them to actually participate in the study. In addition, where possible the Speech and Language Pathologist could assist in facilitating the participation of subjects who have certain specific communication impairments which might otherwise preclude them from participating.

\section{Timing of subject recruitment}

Many patients who are admitted to a rehabilitation medicine inpatient unit have had serious, and sometimes devastating and life-altering, injuries or medical catastrophes. Many of these patients need time to readjust to their new station in life, and following an injury such as spinal cord trauma, there is often a certain amount of grieving that occurs for a part of the patient's life that may be lost to them forever.
Within this setting, many subjects may be hesitant to agree to participate in research projects as they are still trying to cope with the new onset of a significant disability. With time to readjust to the situation, many patients come to view things differently, and go on to become willing volunteers after they have had some time to heal both physically and emotionally. Therefore, whenever possible, the researcher should take this into account, and should try and approach the patient only after they have had time to adjust to their new disability.

In fact, this adjustment may not occur while the person is an inpatient (and sometimes, unfortunately, not at all), and if this is the case, their participation in a potentially time-consuming research protocol should probably not be sought. This must be determined on an individual basis, and may require discussion and consultation with the patient's physician, the therapists involved, the patient's family, and, whenever appropriate, the patient themselves.

Another area where timing of research participation might be an issue is in the TBI population. Because patients with a brain injury often have complex cognitive and behavioral deficits, they generally require in depth and ongoing assessment of these issues. This assessment can be very involved and complex and may take several weeks or months, and the results obtained may have an impact in terms of deciding whether or not the patient might be an appropriate candidate for a research study.

However, many research studies and protocols require the participation of the subject during their entire length of stay on the rehabilitation ward, and some can continue after the patient has been discharged back to the community. Therefore, subject recruitment for a particular study may be necessary before the completion (and sometimes even the initiation) of in-depth cognitive and behavioral assessments. The patient might thus be recruited to participate before it is clear that they have the ability to consent properly to such participation. There must be a balance between the needs of the researchers to gather data throughout the patient's stay and the right of the patient to be protected from participating in a study when they do not fully understand the risks and benefits of the research in question. If there are concerns about decision-making capacity, possible recruitment should be delayed until at least an initial evaluation of capacity can be completed to ensure that subjects are capable of consenting to participate.

\section{The potential for overuse of individual subjects}

The potential exists for individual subjects to be recruited for multiple different studies at various times and thus share a disproportionate burden for research participa- 
tion. This can be a concern especially in two particular situations in rehabilitation medicine. The first is the situation where patients are residents of a particular facility for a long period of time and the second is when they are members of a diagnostic group with relatively small numbers of potential research subjects.

Although a large percentage of rehabilitation patients will have a fairly short stay at an inpatient facility (weeks to months), a smaller subset may be there for a longer period of time, sometimes months to years. Some rehabilitation programs may include long-term care beds where the patient may be there for several years. When patients are residents in a facility for a longer period of time, they may be asked to participate in numerous research projects undertaken by the same, or different, investigator(s) during their stay. The longer the patient stays at that facility, the more likely this is to occur.

Based on the ethical principle of justice, which examines the distribution of costs and benefits of living in a society, no one group should shoulder a disproportionate burden of participation in research. For patients whose stay exceeds a certain period of time, it might be reasonable to have guidelines in place so that these patients will only be asked to participate in a certain number of research projects, perhaps one per year or one every two years, to ensure they do not take on an excessive burden in this area.

Within some diagnostic categories seen by the rehabilitation medicine practitioner, there are relatively small patient numbers. One example is the spinal cord injured population. The incidence of spinal cord injury is approximately 4 per 100000 per year [10], whereas the rate of stroke is approximately 200 per 100000 per year or higher [11]. This means that there are far fewer spinal cord injured patients available to participate in research studies, and as a result the potential for subject overuse is quite high in a population such as this. Many spinal cord injured patients may be asked to participate in research studies on the acute care neurosurgical ward, on the rehabilitation inpatient ward and later on after they have returned to the community. Therefore, patients such as these are at risk of agreeing to participate in a disproportionate number of studies when compared to patients in other diagnostic categories, and of assuming an excessive burden of research participation.

\section{Hope for a cure}

When a person becomes acutely ill, they depend on their medical practitioner to arrive at a correct diagnosis and formulate a treatment program that will restore them to their previous state of health. Unfortunately, there are some situations and diseases where no curative treatment is currently available. This can be devastating information for the patient and their family to receive, especially if the illness or injury is severe or debilitating.

Within the diagnostic groups treated by the rehabilitation specialist, there are many patients where curative treatment is not available, and one can only try to manage the symptoms and maximize functional ability. This includes, but is not limited to, patients with spinal cord injuries and multiple sclerosis.

The relatively recent occurrence of a spinal cord injury in a well-known American movie actor has contributed to an increased focus on research and the development of new treatment options in this area. This has attracted increased funding to assist those involved in spinal cord injury research and has also contributed to the entrance of more scientists to this particular field. Public statements and appearances by this actor have given hope to many individuals who find themselves in similar situations, and who previously may have held out no real hope for a cure. Obviously, these are all very positive developments for patients who have had an injury to their spinal cord.

However, there remains much work to be done in this area, and although progress has certainly been made, we are not yet necessarily on the cusp of a cure for spinal cord injury. This is not always made clear to patients, who may have been waiting a long time for even a small amount of hope that they might someday walk again or regain the use of their hands. When these patients are given the opportunity to participate in a research trial, no matter what the specific content of the trial, they may associate the word "research" with "treatment" or "cure", even when they may be told this is not the case.

There is therefore likely to be a greater probability of therapeutic misconception in this group, because they have suffered a devastating injury with no currently available treatment, and because their hopes have been greatly raised, fairly recently, for the prospect of a cure. The researcher working with this population must be well aware of this, and must endeavor to ensure that patients truly understand the nature and potential outcomes of the trial before they agree to consent to participate.

Patients with MS similarly have been faced with the diagnosis of a neurological disease for which there is no cure. Although symptom management can be reasonably effective with this disease, life expectancy is reduced, and the rate of disability and handicap is higher then in the background population. Recently, the development of medications which modulate the immune system, such as interferons, has shown promise in certain subcategories of the disease [12]. However, the results show a decreased 
number and severity of relapses of the disease, and not a cure or total remission. Many patients with MS have difficulty grasping these concepts, and have become desperate for even a small amount of hope.

These patients, as well, are likely to agree to participate in just about any type of research in the hope that it will provide some degree of benefit for them, even if they are told this is not likely to be the case. As with spinal cord injured patients, they often ask every time they come to the outpatient clinic whether or not there are any research projects under way that they can volunteer for, without any prompting by the physician. Obviously, the potential for therapeutic misconception is also quite high with this group, and this needs to be considered and addressed by the researcher.

\section{Nature of the health care provider-research subject rela- tionship}

As discussed previously, many patients in the rehabilitation medicine setting have recently had fairly serious injuries or illnesses which have had a profound effect on their lives and personal relationships. Within this setting, the bond that develops between these patients and their health care providers (such as physicians, nurses, and therapists) can be exceptionally strong and powerful. Initially, the patient may be totally dependent on the members of the care team for their self-care and bodily integrity, and there is often a component of deep emotional appreciation and gratitude for the care provided during this period. As well, these patients are often on the inpatient ward for a significant period of time, and the relationship they have with members of their care team often deepens and grows during this time.

Although patients in an acute care setting may experience many of these feelings towards their health care providers, it is often not as deep or as intense as the situation on the rehabilitation medicine ward. In this setting, patients may be more likely to agree to participate in a research project out of a desire to please their health care team and because they may perceive their participation as being one way they can "repay" the kindness and care given to them by the members of the team.

This means that the researcher must be especially careful to ensure that the patient understands that their participation is entirely voluntary and that their refusal will not in any way impact not just the quality of their health care, but also their relationship with the members of their care team. The researcher must ensure that the patient understands they have every right to refuse to participate without explanation and that they are free to withdraw from the study at any point in time. Only after the researcher is completely confident that these requirements have been met should the patient be allowed to participate in the study.

\section{Summary}

Various patient populations tend to present unique ethical issues and challenges to those doing research in these areas. Many of these issues have been well discussed in specialties such as pediatrics, obstetrics and medical genetics. Although these challenges also exist in the field of rehabilitation medicine, they have not been previously defined or discussed.

This paper has attempted to identify areas of specific concern when designing research protocols and recruiting subjects for participation in studies in the rehabilitation medicine setting. In some cases, recommendations are provided to address these issues and concerns. It is clear that more work and thought is required in this area to further clarify these ethical issues and to ensure that the rights of rehabilitation patients who participate in research are recognized and respected.

\section{Competing interests}

None declared.

\section{Author's contributions}

Dr. Blackmer was responsible for identifying the topic, reviewing the literature and preparing the manuscript, as well as making applicable revisions.

\section{Acknowledgements}

The author wishes to thank Dr. Barbara Secker for her thoughtful comments and constructive criticisms on the topic.

\section{References}

I. Harrison C, Kenny NP, Sidarous M and Rowell M: Involving children in medical decisions CMAJ 1997, 156:825-828.

2. Annas G]: Protecting the liberty of pregnant patients NEJM 1987, 316:1213-14

3. Kolder VEB, Gallagher J and Parsons MT: Court-ordered obstetrical interventions NEJM 1987, 316: I I92-II 96.

4. Lippman A: Prenatal diagnosis: Reproductive choice? Reproductive control? In: The future of human reproduction Edited by: Overall C. Toronto, The Women's Press; 1989:182-194.

5. Burgess MM, Laberge CM and Knoppers BM: Ethics and genetics in medicine $C M A]$ | $998,158: 1309-1313$.

6. Etchells E, Sharpe G, Walsh P, Williams JR and Singer PA: Consent CMAJ 1996, I55: I77-I80.

7. Roth EJ and Harvey RL: Rehabilitation of stroke syndromes In: Physical Medicine and Rehabilitation 2nd edition. Edited by: Braddom RL. Philadelphia, WB Saunders Co; 2000: III8.

8. Filley CM, Heaton RK, Nelson LM, Burks JS and Franklin GM: A comparison of dementia in Alzheimer's disease and multiple sclerosis Arch Neurol 1989, 46:157-161.

9. Mortimer JA, Pirozzolo FJ, Hansch EC and Webster DD: Relationship of motor symptoms to intellectual deficits in Parkinson's disease Neurology 1982, 32:133-137.

10. DeVivo MJ, Fine PR, Maetz HM and Stover SL: Prevalence of spinal cord injury: re-estimation employing life table techniques Arch Neurol 1980, 37:707-8.

II. Wolf PA, Kannez WB and Venter J: Current status of risk factors for stroke Neurol Clin 1983, I:317-343.

12. Shapiro RT: Medications used in the treatment of multiple sclerosis Phys Med Rehabil Clin N Am 1999, 10:437-446. 


\section{Pre-publication history}

The pre-publication history for this paper can be accessed here:

http://www.biomedcentral.com/1472-6939/4/2/prepub

Publish with Bio Med Central and every scientist can read your work free of charge

"BioMed Central will be the most significant development for disseminating the results of biomedical research in our lifetime. " Sir Paul Nurse, Cancer Research UK

Your research papers will be:

- available free of charge to the entire biomedical community

- peer reviewed and published immediately upon acceptance

- cited in PubMed and archived on PubMed Central

- yours - you keep the copyright 